\title{
A pesquisa sobre Liderança no Brasil: publicações no EnANPAD ao longo de 10 anos
}

\author{
The research about leadership in Brazil: publishing in EnANPAD
}

Recebido em 03.07.2013. Aprovado em 02.10. 2013

Avaliado pelo sistema double blind review

\author{
Shalimar Gallon \\ shalimargallon@hotmail.com
}

Programa de Pós-Graduação em Administração / Escola de Administração / UFRGS - Doutoranda - Porto Alegre - RS - Brasil

Betina Magalhães Bitencourt

betina mb@yahoo.com.br

Programa de Pós-Graduação em Administração / Escola de Administração / UFRGS - Doutoranda - Porto Alegre - RS - Brasil

\section{Carolina Freddo Fleck}

carolina.fleck@gmail.com

UNIPAMPA - Campus Santana do Livramento / Professora Adjunta - Bagé - RS - Brasil

\section{Resumo}

As teorias sobre Liderança demonstram a importância de se investigar nas pessoas que ocupam cargos da alta administração uma relação entre o perfil tido como ideal e as atitudes de trabalho destes indivíduos. Encontra-se, de forma geral, um conceito que aborda os líderes como indivíduos ativos, que mobilizam as pessoas para realizar trabalhos adaptativos, incentivando a aprendizagem rápida e a adoção de novos papéis, relacionamentos e valores. A proposta deste trabalho é apresentar uma meta-análise sobre Liderança para identificar como e de que forma essa temática vem sendo tratada nas pesquisas nacionais. Para isso, optou-se pela busca aos anais do Encontro da Associação Nacional de Pós-Graduação e Pesquisa em Administração (EnANPAD) em todas as áreas que o evento engloba. Essa decisão deu-se pelo fato de que apesar de ser um tema ligado mais a comportamento organizacional e gestão de pessoas, outras áreas vem buscando pesquisas que possam auxiliar na identificação do quanto a Liderança pode influenciar nessas. Os trabalhos encontrados demonstraram manter-se em torno de poucos assuntos principais e com pequena contribuição para esta temática. Nota-se também que há uma presença forte de literatura estrangeira ligada às pesquisas mencionadas.

Palavras-chave: Liderança; EnANPAD; Teorias; Meta-análise.

\section{Abstract}

The theories about Leadership demonstrate the importance of investigating on people in positions of top management a relationship between the profile considered ideal and work's attitudes of these individuals. There is, in general, a concept that addresses the leaders as active individuals who mobilize people to do adaptive work, encouraging the rapid learning and adopting new roles, relationships and values. The purpose of this paper is to present a meta-analysis about leadership to identify how and in what way this issue has been dealt with in national researches. For this, we chose to search the annals of the Meeting of the National Association of Graduate Studies and Research in Administration (EnANPAD) in all areas of the event encompasses. This decision was due to the fact that although the subject was related more to organizational behavior and management of people, other areas of research have sought to assist in the identification of how leadership can influence these. The works found demonstrated keep around a few main issues and with small contribution to this theme. We noted also that there is a strong presence of foreign literature linked to research mentioned.

Keywords: Leadership; EnANPAD; Theories; Meta-analysis. 


\section{A pesquisa sobre Liderança no Brasil: publicações no EnANPAD ao longo de 10 anos}

\section{Introdução}

A temática liderança vem sendo alvo de pesquisas há muitas décadas e conforme afirma Hall (2004), inicialmente com uma tendência a romancear o tema e a enfatizar que os novos líderes deveriam ser os "salvadores" das organizações, como se houvesse no indivíduo com aptidão para liderar uma aura que o torna mágico e capaz de solucionar os mais complexos problemas organizacionais e tornar a menor das empresas na mais eficaz e lucrativa para o seu dono. No entanto, a realidade das organizações e a quantidade de pesquisas que surgiram com a popularização do tema demonstraram que nem sempre o líder pode "salvar" a organização.

Seguindo essa lógica, surgiram as pesquisas sobre perfis de liderança e uma lógica de adequação destes perfis ao tipo de organização em que se trabalha, ou seja, a cada nova pesquisa mudaram os argumentos, surgindo novos vieses, mas não se chegou a um ou alguns denominador(es) comum(ns) para o que é liderança e como esta deve ser tratada nas organizações. As conceituações são diversas e depende da percepção de cada pesquisador a escolha do enfoque (BENNIS, 1996; VENDRAMINI, 2000; HALL, 2004).

As pesquisas sobre liderança são apresentadas como oriundas já do tempo dos escritos em papiro, nos quais se apontava a importância de um líder para as civilizações. Com o advento das teorias administrativas e a indicação da necessidade do líder nas organizações surgiram diferentes enfoques para tentar explicar essa necessidade da figura de alguém acima dos demais nas organizações e que deve conduzir os processos para 0 sucesso organizacional (AZEVEDO, 2002). E justamente por existirem essas diferentes linhas e controvérsias entre as pesquisas, inclusive a grande diversidade de definições racionais e subjetivas, introduzidas nos aspectos sob o qual a temática se apresenta, questiona-se, segundo Klann e Cartwright (2004), se esta vem a ser mais arte ou mais ciência?

Estes autores concluíram que a Liderança pode caracterizar-se como uma combinação das duas coisas: ciência e arte. É considerada ciência visto que é reconhecida no âmbito acadêmico, além de ser composta de regras e princípios. Porém, estas regras e princípios devem ser aplicados de maneira criativa, fato que a torna, também, uma forma de arte (KLANN; CARTWRIGHT, 2004). Com isso, os pesquisadores perpassam diferentes correntes teóricas para seu posicionamento. Contudo, ainda não se tem uma real noção de como está a situação no meio acadêmico brasileiro. Sabe-se que

os estudos clássicos da liderança baseavam-se nas grandes personalidades de cada época, retratando-os como figuras heróicas. Sob a ótica de alguns pensadores, as massas aguardavam ansiosamente por uma voz ativa que as comandasse. O líder possuía características natas, o que lhe garantia o status e o poder diante dessas massas (VENDRAMINI, 2000, p. 26).

Mantendo a ideia de que o líder é quem toma as rédeas da organização e a faz crescer, enfatiza-se uma maior perspectiva da importância às investigações sobre as pessoas que ocupam cargos da alta administração, investigando uma relação entre o perfil de líder tido como ideal e as atitudes de trabalho destes indivíduos. Encontra-se, de forma geral, nos trabalhos relacionados a este objetivo um conceito que aborda os líderes como indivíduos ativos, que mobilizam as pessoas para realizar trabalhos adaptativos, incentivando a aprendizagem rápida e a adoção de novos papéis, relacionamentos e valores.

Com o intuito de contribuir para o escopo das pesquisas na temática liderança, o objetivo deste estudo foi realizar um levantamento dos artigos publicados em um dos principais eventos da área de Administração no Brasil, que abordam a temática sobre Liderança. O propósito da pesquisa foi realizar um levantamento sobre as abordagens que mais se destacam, especialmente verificando se existem temas que parecem emergir por 
"moda" ou se as linhas que surgiram ao longo das décadas se mantêm em virtude das diferentes perspectivas dos pesquisadores da área.

Para tal, definiu-se como método de pesquisa a meta-análise, que se apresentou como melhor opção pela possibilidade de um estudo sistemático com característica longitudinal, permitindo a comparação das informações ao longo dos anos. Existe uma crescente tendência nas ciências administrativas por pesquisas que utilizem meta-análise/revisão sistemática. Estas pesquisas permitem que se construa um histórico em torno de referenciais já utilizados e que até então não se tem domínio de como e com base em que corrente(s) teórica(s) e/ou metodológica(s) se sustentam. Considera-se também por esta razão importante a meta-análise sobre publicações na temática da liderança.

Os dados coletados foram primeiramente tabulados e categorizados, utilizando-se em seguida uma análise descritiva para os mesmos. De forma a apresentar detalhadamente o estudo, esse se divide em outras três partes, além desta introdução. 0 tópico a seguir apresenta o método que norteou o estudo; seguido de um tópico com a análise realizada com a base de dados coletados, evidenciando suas principais características, seguido então das considerações finais a respeito do tema. Ressalta-se que sendo uma meta-análise as considerações com relação ao referencial teórico encontram-se vinculadas aos tópicos de análise e considerações finais.

\section{Método utilizado na pesquisa}

O método desta pesquisa fundamenta-se em uma meta-análise, a qual se baseia em um método quantitativo que busca analisar os resultados de estudos anteriormente realizados, geralmente extraídos de trabalhos publicados, e sintetizar as suas considerações ou extrair novas considerações (LUIZ, 2002). Para levantamento dos dados, buscaram-se os artigos do Encontro da Associação Nacional de Pós-Graduação e Pesquisa em Administração (EnANPAD), pois além de ser o principal evento da área de Administração do Brasil, possui também reconhecimento na América Latina.

A Associação Nacional de Pós-Graduação e Pesquisa em Administração (ANPAD) é responsável pela organização do EnANPAD, que congrega docentes, pesquisadores, alunos e profissionais das ciências administrativas e áreas correlatas. Trata-se de um espaço para apresentação e debate de trabalhos acadêmicos de diferentes áreas temáticas e de questões contemporâneas da Administração. Além disso, considera-se 0 EnANPAD como uma "porta de entrada" aos artigos acadêmicos de relevância nacional, pois neste evento os trabalhos selecionados são apresentados e debatidos para posteriormente serem publicados em periódicos científicos da área.

Sendo o objetivo do artigo, identificar como e de que forma a temática Liderança vem sendo tratada nas pesquisas nacionais, optou-se pela busca aos anais do EnANPAD em todas as áreas que o evento engloba. Essa decisão deu-se pelo fato de que apesar de ser um tema ligado mais a comportamento organizacional e gestão de pessoas, outras áreas vem buscando pesquisas que possam auxiliar na identificação do quanto a Liderança pode influenciar nessas. Na presente pesquisa foram utilizados como base de análise os artigos publicados sobre Liderança no período de 1999 a 2008 nos anais do EnANPAD. Este período foi escolhido, pois permitiu à pesquisa a identificação dos temas mais abordados em um período de fechamento de década (e século) $^{1}$, além de efetivarem-se já duas décadas de pesquisa mais enfática sobre liderança, já que os primeiros estudos datam da década de 1980 (AZEVEDO, 2002); bem como a sequência de trabalhos de praticamente toda a década seguinte demonstrando o crescimento da temática para as ciências administrativas. No total, foram levantados 44 artigos que abordavam a temática Liderança.

\footnotetext{
${ }^{1}$ No momento de coleta de dados, o EnANPAD ainda não havia ocorrido, e consequentemente, seus artigos não estavam disponibilizados. Em função disso, o ano de 2009 não foi utilizado na análise de dados para fechar os dados da primeira década do século XXI.
}

CGE * v. $1 *$ n. 1 * Jan-Abr 2013 * 01-16 


\section{A pesquisa sobre Liderança no Brasil: publicações no EnANPAD ao longo de 10 anos}

A seleção dos artigos obedeceu aos seguintes critérios: (1) estar na faixa de tempo pesquisada, de 1999 a 2008; e (2) ter como foco de pesquisa a Liderança. Para seleção prévia dos artigos buscou-se através da leitura e análise dos títulos e respectivos resumos, identificar esses temas. Posteriormente, os artigos selecionados foram analisados por meio da leitura dos títulos e respectivos resumos, além da sondagem do conteúdo de cada artigo, com o objetivo de classificá-los de acordo com os critérios definidos. No entanto, como alguns artigos abordavam mais de um tema, procurou-se observar se o tema Liderança era o foco do estudo.

A pesquisa se deu através de palavras-chave (liderança - líder - estilo(s) de gestão) no sistema de busca do CD-ROM dos anais do evento, ano a ano. Destaca-se que no período de 1999 a 2003 a busca foi realizada apenas nas palavras-chave e resumo - pois o software restringia a pesquisa a estes tópicos, já demonstrando que os primeiros anos da virada da década permitiram também o melhoramento da busca por materiais de diferentes temáticas. Já a partir de 2004 a busca passou a ser permitida também no conteúdo do trabalho, com o melhoramento do software dos anais do evento. No entanto, apesar de essa busca mais refinada nos últimos cinco anos - do período de análise - os dados coletados ainda passaram por um filtro das autoras, no qual foram retirados os artigos que não analisavam realmente a temática abordada neste estudo.

Em relação à classificação da abordagem e da estratégia de pesquisa dos artigos analisados foram utilizados três parâmetros. Primeiramente, analisaram-se as informações sobre os métodos apresentados nos próprios artigos, a fim de preservar as nomenclaturas abordadas pelos autores dos artigos em questão. Quando não havia dados explicitados pelos autores, utilizou-se da interpretação das tipologias apresentadas de forma implícita. E, por último, foi criada uma classificação do tipo não informada, para agrupar os estudos desprovidos de nomeação ou que não tinham características implícitas satisfatórias para a classificação dentro de alguma abordagem ou método (GODOI; BALSINI, 2006).

A análise dos dados foi realizada de forma descritiva, mesclando contagem de percentuais, como no caso das instituições de origem e dos autores e uma análise mais voltada ao conteúdo das respostas, com contagem das mais mencionadas, no que diz respeito aos métodos utilizados e às teorias abordadas. Na sequência apresentam-se os resultados encontrados e o perfil das pesquisas sobre liderança no EnANPAD.

\section{Os estudos sobre Liderança na última década}

O primeiro levantamento realizado na pesquisa diz respeito ao quantitativo de artigos apresentados em cada ano pesquisado nos anais do evento, na temática Liderança, entre os anos de 1999 e 2008 . Foi encontrado um total de 44 artigos específicos sobre o tema (destacando que inicialmente foi realizado o filtro a partir das características apresentadas no tópico anterior), distribuídos da seguinte forma: nos primeiros dois anos (1999 e 2000) apenas dois artigos foram publicados em cada ano; entre 2001 e 2002 foram três por ano; de 2003 a 2006 foram publicados quatro artigos por ano, com exceção de 2005 quando foram cinco publicados. 0 ano de 2007 apresentou alta na publicação, com dez artigos, e em 2008 foram sete publicados.

Com isso, pode-se observar que há um aumento considerável no número de pesquisas com enfoque na Liderança, que isoladamente significam no mínimo ampliação do número de pesquisadores sobre o tema e poderia significar que a temática passou a ter maior relevância na seleção de artigos para o evento. No entanto, essa realidade não necessariamente significa um desenvolvimento do tema em questão, primeiramente porque o tipo de seleção dos artigos para o evento não pressupõe que quanto maior o número de artigos para uma temática, maiores serão as futuras publicações. A publicação depende da qualidade dos trabalhos e da blindreview realizada, que pode ou não indicar mais artigos de uma ou outra temática. Além disso, os resultados sobre o tema demonstraram que ainda há uma grande dispersão na concentração de focos nos artigos. 


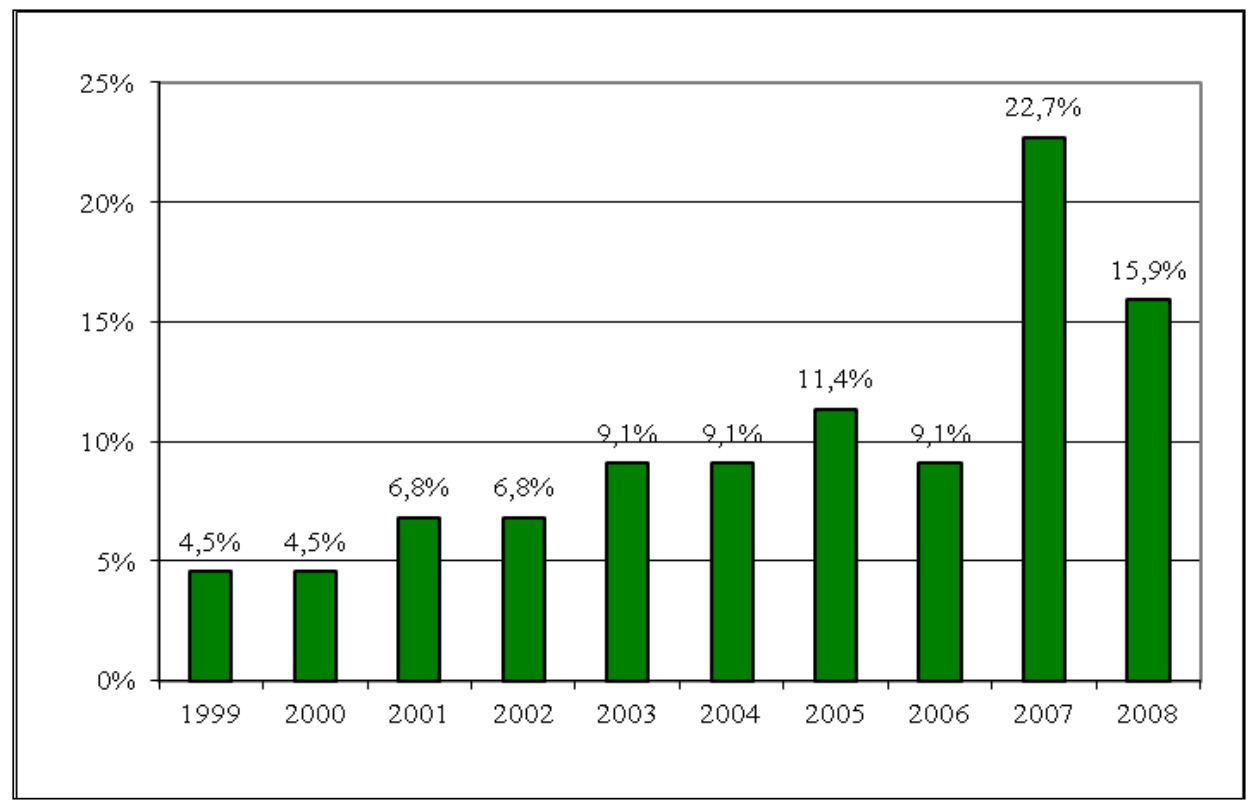

Gráfico 1 - Percentual de artigos publicados a cada ano em relação ao total de artigos

Considerando em um segundo momento os autores dos artigos sobre a temática, foi possível verificar que os pesquisadores variam e não há um grupo que mantém constante publicação ao longo da década analisada. Apesar de serem encontrados nomes recorrentes, para os fins dessa pesquisa foi considerado somente 0 primeiro autor de cada artigo, fato que acabou limitando a análise desses dados, mas que se considerou pertinente pela tendência que se conhece (do senso comum acadêmico) de que os primeiros autores tendem a serem os principais pesquisadores. Entre os autores que publicaram artigos nos últimos dez anos, puderam ser destacados, como primeiros autores, os nomes de Paulo Sérgio Altman Ferreira e Vânia Maria Jorge Nassif, com duas publicações cada.

Além dos autores, foram identificadas suas instituições de origem, procurando mapear se haveria algum destaque nesse sentido, indicando uma tendência de tal instituição de valorizar a temática nas pesquisas realizadas. Entre as universidades encontradas nos 44 artigos, destaca-se a Mackenzie, com seis artigos, seguida da UFBA e UFPE com três artigos cada e das instituições de ensino superior FEAD, UEL, UFMG, UFRGS e UnB com dois artigos cada, ao longo da década pesquisada. Esses dados demonstram não haver uma única universidade alinhada com a temática da liderança e uma pulverização do tema entre as instituições de diferentes regiões do país.

Buscou-se identificar entre os métodos utilizados se haveria ou não uma tendência de pesquisas com um mesmo método empregado para investigar a temática liderança. Um dos propósitos de tal investigação foi o de identificar se as pesquisas na temática ainda seriam de caráter mais exploratório, pois como afirma Hair (2005), neste caso haveria mais estudos qualitativos, ou se as pesquisas se consolidam em estudos de caráter descritivo, no qual a abordagem pode variar entre qualitativa e quantitativa permitindo explorar públicos maiores com a coleta de dados quantitativos.

Primeiramente foram analisadas as estratégias de pesquisa adotadas, a partir das quais se pode observar uma predominância de estudos de caso (50\%), sendo que $27 \%$ são estudos de caso único e $23 \%$ estudos de caso múltiplo. Também se pode observar uma grande quantidade de artigos que não informam a estratégia utilizada $(15,9 \%)$, conforme mostra o Gráfico 2. Além do Estudo de Caso, destacam-se estratégias de pesquisa como os Ensaios Teóricos; Pesquisa-Ação e Fenomenologia, todos de abordagem qualitativa. As surveys e estudos de validação de instrumentos, que correspondem a estratégias quantitativas aparecem em menor quantidade se 


\section{A pesquisa sobre Liderança no Brasil: publicações no EnANPAD ao longo de 10 anos}

comparada ao total de estratégias qualitativas apresentadas (mesmo que no gráfico survey apareça na frente de estudos de fenomenologia, por exemplo).

Considerando a afirmação de Hair (2005) a respeito da tendência de abordagem qualitativa para estudos exploratórios e abordagem quantitativa em estudos descritivos, pode-se argumentar que a temática liderança nas pesquisas nacionais está em uma fase ainda exploratória, passando para alguns estudos descritivos. Mesmo que as temáticas se repitam, como será apresentado na sequência, há uma indicação de que esta repetição pode ser uma tendência de buscar compreender quais as teorias que realmente convergem com a realidade das organizações brasileiras e podem ser consideradas pertinentes para linhas de pesquisa futuras.

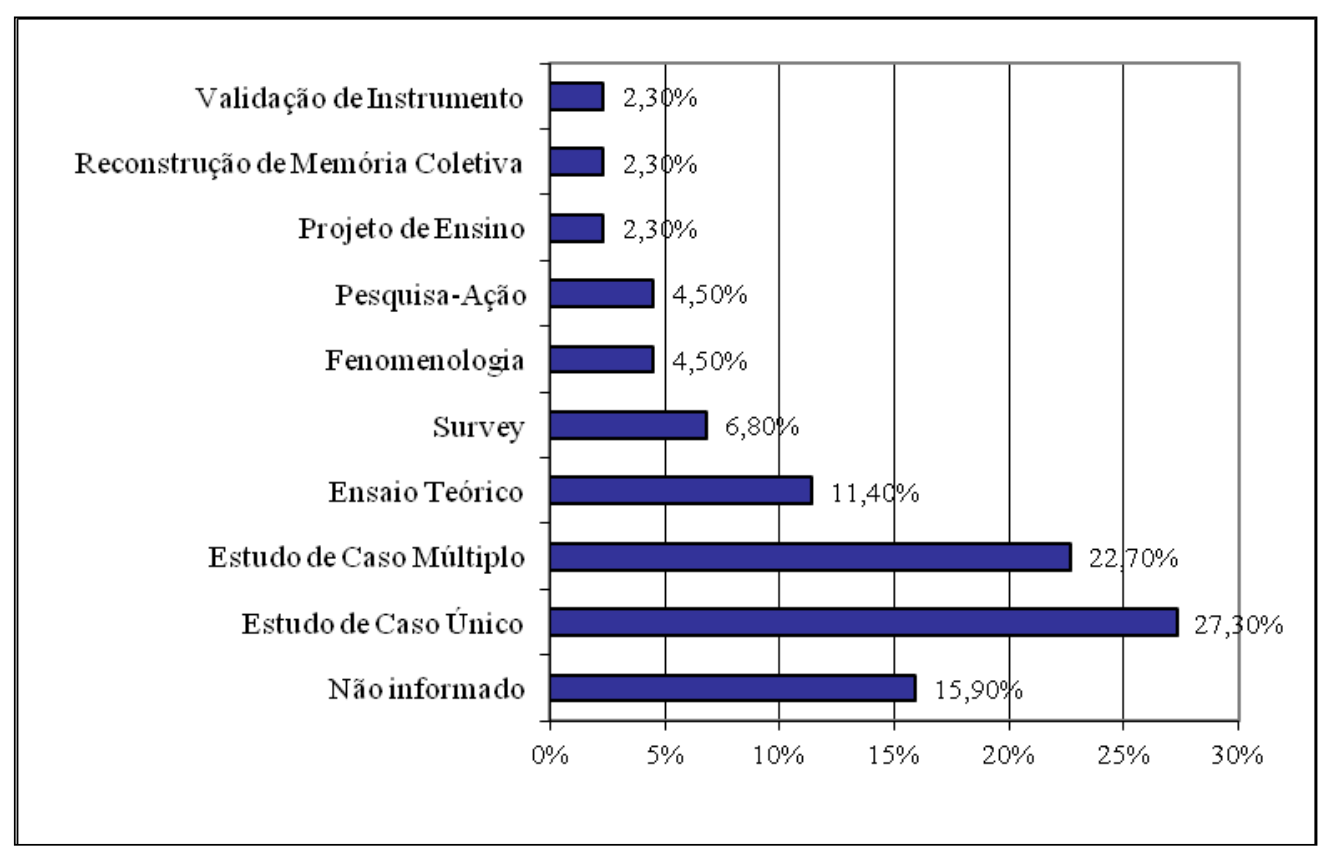

Gráfico2 - Estratégia de Pesquisa

Os dados do Gráfico 3 complementam essa análise ao demonstrar que a soma de estudos qualitativos, ensaios teóricos e de abordagem mista chega a $62,8 \%$ dos artigos publicados. Esse total indica, apesar de não haver a informação explícita nos artigos, estudos de caráter exploratório, ou seja, estudos que poderão dar embasamento para o crescimento da temática. Os estudos quantitativos, que correspondem a $31,8 \%$ dos artigos publicados tendem a demonstrar pesquisas de caráter descritivo (HAIR, 2005), no entanto, com o total apresentado podem ser parte desta lógica ainda exploratória, com avanços de pesquisa, como as validações de instrumentos de coleta de dados como forma de reforçar as questões de liderança na área de Administração. Em uma análise da distribuição das abordagens, verificou-se que os estudos quantitativos encontram-se publicados ao longo da década, sem uma concentração específica em um ou dois anos, indicando que ainda são incipientes e corroborando a tendência de estudos mais exploratórios vinculados à temática. 


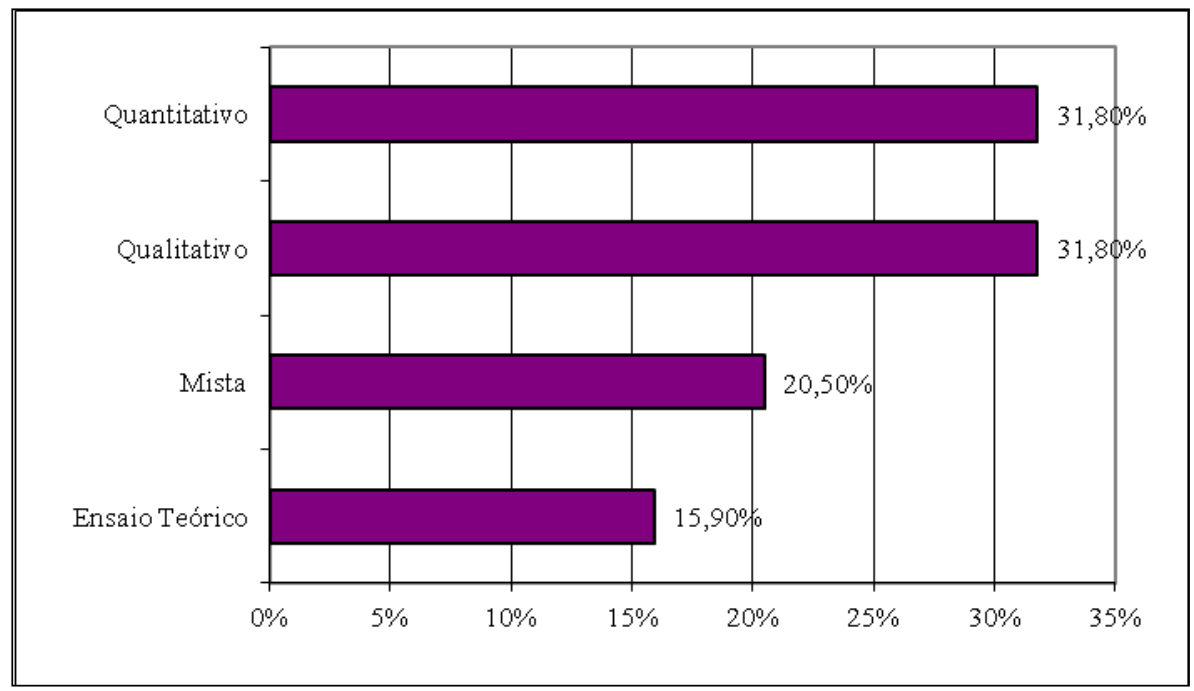

Gráfico 3 - Abordagem

Considerando as áreas temáticas do evento em que foram publicados artigos sobre Liderança, identificou-se que o tópico é explorado em diferentes áreas, indicando diferentes enfoques de pesquisa e tentativa de incluir as questões de liderança nos mais variados aspectos das pesquisas da área administrativa. Como se imaginava as áreas de Gestão de Pessoas e Relações de Trabalho e Estudos Organizacionais foram as que mais apresentaram concentração de artigos, com $71 \%$ do total encontrado. Os $29 \%$ restantes ficaram divididos entre: Gestão Social e Ambiental; Gestão de Ciência, Tecnologia e Inovação; Ensino e Pesquisa em Administração e Contabilidade; Empreendedorismo e Comportamento Empreendedor; Administração da Informação; Administração Estratégica; e Administração Pública. Possivelmente as diferentes áreas fundamentam-se nos objetos de pesquisa escolhidos para a análise da liderança, considerando que o tema, em termos de teoria, normalmente é vinculado às áreas de Gestão de Pessoas e Estudos Organizacionais.

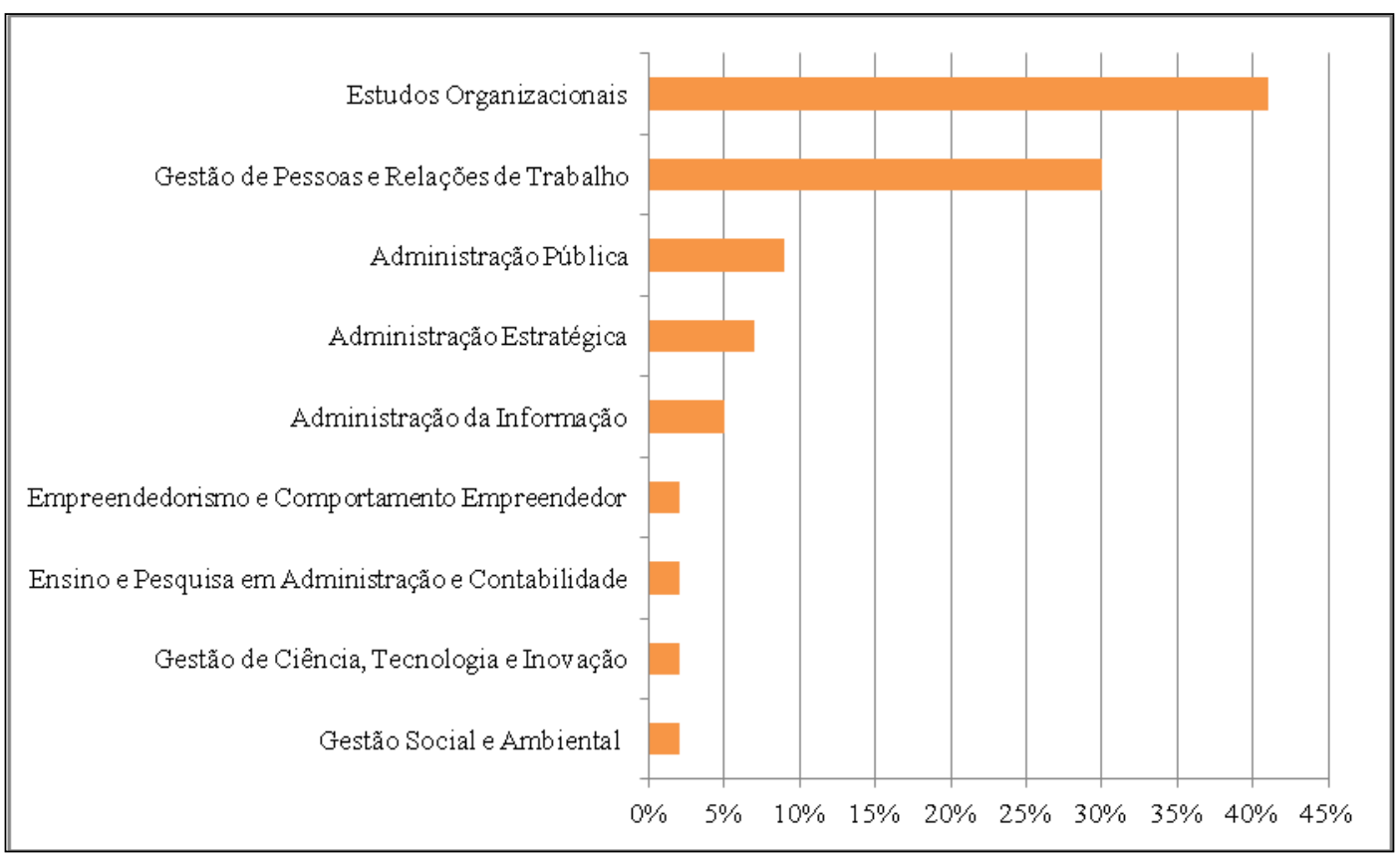

Gráfico4 - Áreas do EnANPAD 


\section{A pesquisa sobre Liderança no Brasil: publicações no EnANPAD ao longo de 10 anos}

Destaca-se que entre as áreas temáticas encontradas algumas já não existem e outras mudaram a nomenclatura, apenas o que muda são os anos em que se utiliza uma ou outra, sendo assim, consideraram-se as nomenclaturas mais atuais para a finalização do artigo.

\section{Análise do Foco Temático dos Artigos}

Após uma primeira análise dos métodos utilizados e das principais áreas com publicações sobre Liderança passou-se a realizar a análise dos subtemas mais destacados. Considerando os 44 artigos que tinham como ênfase as teorias de liderança e os tópicos abordados foram classificados cinco grandes grupos temáticos para melhor analisá-los. Os grupos foram determinados pelos assuntos mais pesquisados e ficaram divididos em: Perfil e Estilos, Competências, Desenvolvimento de Liderança, Papel do Líder e Outros. Pelos grupos temáticos, observa-se que o Papel do Líder e Perfil e Estilos foram os grupos com maior frequência, como pode ser visualizado no Quadro 1.

\begin{tabular}{|c|c|c|c|c|}
\hline Perfil \& Estilos & Competências & $\begin{array}{c}\text { Desenvolvimento de } \\
\text { Liderança }\end{array}$ & Papel do Líder & Outros \\
\hline $\begin{array}{l}\text { - Estilo de Liderança } \\
\text { - Gestão do Conhecimento } \\
\text { - Liderança Carismática } \\
\text { - Liderança Criativa } \\
\text { - Liderança Situacional } \\
\text { - Liderança } \\
\text { Transformacional e } \\
\text { Transacional } \\
\text { - Perfil do Líder } \\
\text { - Produtividade }\end{array}$ & $\begin{array}{l}\text { - Aprendizagem } \\
\text { - Competências } \\
\text { - Controle Afetivo } \\
\text { - Discurso do Líder } \\
\text { - Empreendedorismo }\end{array}$ & $\begin{array}{l}\text { - Construção de } \\
\text { Identidade } \\
\text { - Desenvolvimento do } \\
\text { Líder }\end{array}$ & $\begin{array}{l}\text { - Coordenação de práticas } \\
\text { - Cultura } \\
\text { - Desenvolvimento de RH } \\
\text { - Gerenciamento de Impressão } \\
\text { - Gestão da Confiança } \\
\text { - Gestão da Criatividade } \\
\text { - Gestão de Pessoas } \\
\text { - Gestão de Stakeholders } \\
\text { - Influência do Líder } \\
\text { - Motivação } \\
\text { - Papel do Líder }\end{array}$ & $\begin{array}{l}\text { - Conceito de } \\
\text { Liderança } \\
\text { - Poder } \\
\text { - Processo de } \\
\text { Liderança }\end{array}$ \\
\hline 14 artigos & 9 artigos & 3 artigos & 14 artigos & 4 artigos \\
\hline
\end{tabular}

A partir dos cinco grupos temáticos, os subtemas foram categorizados para proceder a análise do conteúdo dos artigos, procurando investigar as principais teorias utilizadas e conclusões apresentadas para a temática. Estas análises encontram-se divididas em tópicos a seguir, considerando os grupos temáticos levantados para a análise. 


\section{Perfil \& Estilos de Liderança}

Os artigos pesquisados que abordam Perfil e Estilos de Liderança somaram 14 publicações no decorrer dos dez anos analisados. Os temas contemplados nestes artigos são o próprio Perfil do Líder, os Estilos de Liderança, como Liderança Carismática, Liderança Criativa, Liderança Situacional e Liderança Transformacional e Transacional, além da Gestão do Conhecimento e a Produtividade, ligadas aos traços dos líderes nas organizações.

Dentre as abordagens metodológicas mencionadas, destacam-se os estudos de caso (únicos e múltiplos) e revisão teórica, porém essa última mostra-se com bem menos expressão nestes assuntos pesquisados. Ainda, entre os autores mais citados nos artigos, destacam-se Bennis (1988; 1995; 1996; 1999a; 1999b; 2001) e Rickards e Moger (1999a; 1999b; 2000), seguidos de Burns (1978; 1979), Bryman (1991; 1992; 2004) e Yukl (1989; 1996; 2002).

O perfil do líder foi associado ao desempenho das organizações, como no item produtividade, conforme citado em um dos artigos. Neste estudo, dos indicadores de produtividade pesquisados, as variáveis que fundamentam a liderança com base nas características pessoais do líder foram as que se associaram com as unidades que obtiveram melhores indicadores de qualidade. 0 estudo conclui que é necessário que ocorra uma congruência entre a percepção do gerente e dos geridos no quesito produtividade/qualidade. A liderança, portanto, é vista como um diferencial competitivo no mercado, pois o estilo de liderar reflete sobre o desempenho dos seguidores e também no sucesso das organizações.

Os tipos de liderança também foram estudados a partir da sua relação com a identidade cultural, com a gestão social das organizações, com o comprometimento afetivo dos funcionários para com a empresa, com a gestão da diversidade nas organizações, com a gestão do conhecimento, e até na utilização de mentoria nas universidades.

Entre os estudos, pode-se destacar a discussão sobre o gênero do líder e a sua influência no estilo de liderança, pois, enquanto alguns autores, como Bartol (1978) e Engen et. al, (2001), defendem a ideia de que há pouca ou nenhuma influência do gênero nos estilos de liderança, há autores que afirmam o contrário. É o caso das pesquisas de Bass e Avolio (1993) e Bass (1996), que afirmam existir diferenças entre os estilos de liderança, sendo que o estilo transformacional predominaria nas mulheres por serem caracterizadas como pessoas mais abertas e encorajadoras de maior participação nas tomadas de decisões, o que poderia acarretar em liderados mais motivados e de melhor desempenho. No entanto, a pesquisa encontrada nesta seção revela que não foram encontradas diferenças significativas entre gêneros e os estilos de liderança, embora tenha sido utilizado 0 mesmo instrumento de Bass e Avolio (1993).

Em relação ao perfil e estilos de liderança, percebe-se que não há predominância de pesquisas em algum estilo, tampouco em um determinado tema, embora a quantidade de artigos encontrados não sirva para tomar isso como uma verdade única. É importante salientar, ainda, que por se tratar de artigos em sua maioria em formato de estudos de caso, não há possibilidade de generalização dos resultados, sendo que a reaplicação em contextos diferentes poderá trazer também resultados diferentes.

\section{Competências}

Os artigos que abordaram temas referentes às Competências do Líder nas organizações totalizaram nove estudos. A partir disso foram levantados os assuntos subjacentes a esse tema, tais como: aprendizagem, comunicação, controle afetivo, democracia, desempenho, emoção, empreendedorismo, gestão pública, identificação com o líder, inovação, liderança de equipes, mitologia, perfil do gestor, poder e teoria organizacional. Além disso, apenas dois artigos utilizaram determinados tipos de liderança, tais como a Abordagem dos Traços e a Situacional, Liderança Criativa e a Teoria Comportamental e Contemporânea, para explicar as competências dos líderes. Os outros estudos abordaram a liderança de modo geral. Apesar de a grande diversidade de autores utilizados para embasar esses estudos de Liderança, os autores mais citados 


\section{A pesquisa sobre Liderança no Brasil: publicações no EnANPAD ao longo de 10 anos}

foram: Bass e Avolio (1990; 1993; 1994; 1995), Bergamini (1994), Hersey e Blanchard (1986), Kotter (1992; 2000) e Rickards e Moger (1999b; 2000).

Em muitos estudos observou-se que o conceito de competência é percebido como Conhecimento, Habilidades e Atitudes (CHA). Embora autores como Le Bortef (2003) e Zarifian (2001) apontam que a competência está relacionada com fatores individuais, eles observam que a competência também está relacionada com o valor agregado e a entrega. Zarifian (2001, p. 42) também ressalta que a competência profissional está relacionada com 0 posicionamento frente aos eventos, que são "os novos problemas colocados pelo ambiente, que mobilizam a atividade de inovação".

Dentre as pesquisas realizadas, dois estudos enfatizaram que líderes criativos têm maior capacidade estratégica, assim também como a empreendedora. Já outro artigo observa que uma liderança voltada para 0 relacionamento, favorece a aprendizagem nas organizações. Ainda em relação à liderança criativa e as competências, levantou-se que não há relação ao sexo e nem a idade, embora tenha uma relação positiva com o grau de instrução. Sobre o empreendedor, foi observado que ele busca alinhar suas ações de acordo com 0 ambiente e seus objetivos pessoais e que em determinado momento da sua maturidade pessoal e profissional, ele se sente mais líder do que empreendedor. Entre os estudos levantados, além de uma revisão acerca das teorias organizacionais, também foi encontrado um projeto de pesquisa que busca ver a relação entre 0 representante da turma e as competências naturais ao seu desempenho, que são expectativas naturais do profissional formado em administração.

Após a análise dos resultados percebe-se que a competência do líder está baseada na experiência (fazer, errar e corrigir o comportamento), na comunicação, a qual demanda uma intervenção multidisciplinar, além da competência de fazer parte de equipes e a habilidades de liderança. Outra competência destacada é a de controle sedutor, a qual se baseia em uma técnica sutil e eficaz de controlar o indivíduo pelo afeto e procura fazer com que o empregado se identifique com o seu superior e, assim, haja como tal. É enfatizado que é preciso considerar a subjetividade do sujeito, a qual passa exercer influência no papel de liderança, como por exemplo, a capacidade para se apropriar da emoção e integrá-la na ação gerencial. Também foi observado que algumas das características pessoais fazem parte do repertório individual e que são adquiridas na formação da personalidade, sendo assim, não passíveis de serem aprendidas ou imitadas por outras pessoas. Exemplo disso seria a necessidade de realização, iniciativa, ousadia, afetuosidade, criatividade, introversão ou extroversão. Já em outro estudo é observado que as trocas de percepções/lógicas e vivências que são compartilhadas acabam por tecer uma aprendizagem. Isso pode construir e consolidar os recursos emocionais para o desenvolvimento de competências de liderança.

\section{Desenvolvimento de Liderança}

Os artigos encontrados na pesquisa que versaram como tema central o desenvolvimento de liderança foram três. Os temas visualizados foram o processo de construção da identidade do líder, seguido da aplicação de testes com o objetivo de desenvolver o líder, de maneira a contribuir para uma gestão com foco na liderança e, assim, buscar melhorias no desempenho organizacional como um todo.

Os autores mais citados no embasamento teórico destes estudos foram Bergamini (1994; 2002), Bryman (1992; 1997), Gordon (2000) e Hersey e Blanchard (1969; 1988). Nota-se, entretanto, que não há uma concentração de autores citados neste assunto, pois o restante dos autores mencionados não se repetiu em mais de um artigo.

O primeiro artigo retrata o discurso dos líderes perante a formação de identidade das organizações na mídia e, consequentemente, para a comunidade. É ressaltado que há na elaboração, por parte dos líderes, um modelo organizacional de comunidade ou família, construindo a base para comportamentos e relacionamentos nas organizações. Ou seja, o desenvolvimento do trabalho do líder, e do próprio como representante da instituição, afeta diretamente nos rumos da organização e, dessa forma, a imagem da mesma como um todo.

CGE * v. 1 * n. 1 * Jan-Abr 2013 * 01-16 
Já o segundo estudo aponta as relações entre tipos de poder e autoridade, características pessoais de líderes e liderados, interrelações sociais, poderes atribuídos aos cargos, necessidade de alcançar objetivos corporativos e conjuntos de competências desejadas e necessárias ao seu exercício do papel de líder. Nesse artigo, é analisado um projeto de modelagem das ações da liderança que parte da matriz estrangeira para a subsidiária brasileira. Além de evidenciar a importância do líder na organização, esse estudo mostra que é possível trabalhar no desenvolvimento dos líderes, em vez de confiar no surgimento natural deles, que nem sempre acontece na velocidade, quantidade e com a qualidade desejada, ou demandada pela organização.

Seguindo a linha de instrumentos de desenvolvimento de líderes, o terceiro artigo trata do desenvolvimento e dos testes de uma ferramenta utilizada para a gestão do conhecimento nas organizações. 0 papel do líder na gestão do conhecimento é imprescindível, pois se trata de uma dimensão da gestão nas organizações e é ele quem está próximo das pessoas portadoras do conhecimento nas empresas.

Há, ainda, a ressalva de que os instrumentos utilizados no desenvolvimento dos líderes, bem como na gestão do conhecimento nas organizações devem ser adaptados às peculiaridades de cada instituição, já que cada uma atua sob contextos diferentes e, portanto, têm necessidades e capacidades diferentes.

\section{Papel do Líder}

Os artigos que abordaram temas referentes ao Papel do líder dentro das organizações totalizaram quatorze estudos. Os temas que subsidiaram os estudos eram relacionados com a cultura, comprometimento e desempenho organizacional, os desafios dos líderes na Gestão de Pessoas, gerenciamento de impressão, motivação, poder, interesse, expectativa e confiança dos liderados, gestão da criatividade, processo de aprendizagem, coaching, capital intelectual, desenvolvimento sustentável e estrutura organizativa dos sindicatos. Pode-se observar que a Liderança Carismática, Transformacional, Transacional, Empreendedora, Política e a baseada no modelo Stakeholders foram os tipos de lideranças destacadas nos artigos. Os outros estudos buscaram abordar a Liderança de forma ampla. Para embasar esses estudos, os autores mais citados foram Schein (1991; 1992; 1996) e Ulrich (1997; 1998a; 1998b; 2000). Outros autores também foram destacados, tais como Bass e Avolio (1993), Benis e Nanus (1988), Bergamini (1994; 1997) e Limongi-França e Arellano (2002).

Pode-se observar que cinco artigos destacam o fato de terem sidos realizados como uma pesquisa de estudo de caso único e que esses resultados não poderiam ser generalizados. 0 que se percebe é que estes estudos trazem conclusões pontuais se referindo a casos específicos e seus resultados não vem contribuir teoricamente para os estudos de Liderança. Há uma revisão teórica que também busca somente levantar as teorias existentes. Nota-se que poucos estudos buscam trazer novas teorias, mas sim, apenas constatar o que já foi estudado anteriormente. Embora três artigos tenham se baseado em cálculos estatísticos de maior confiabilidade, seus resultados apontam para o levantamento de necessidades, as quais já foram apontadas em outras pesquisas.

Os resultados encontrados em três artigos apontam que o papel do líder é de influenciar e concretizar a cultura corporativa, embora seja enfatizado que a liderança e o comprometimento organizacional dependem do ramo de atividade e perfil da empresa pesquisada. Outros dois estudos mostram que o comprometimento do liderado está relacionado com o interesse do mesmo quanto a sua carreira e o cargo ocupado. Também é ressaltado que a relação dos liderados com o líder é baseada no respeito, honestidade e crédito no empregado, assim como, também devem identificar os funcionários que melhor se ajustam as necessidades da organização. Percebe-se que o Papel dos líderes dentro das organizações é de desenvolver competências, assim como sensibilizar seus liderados e possibilitar que alcancem seus objetivos.

Um questionamento apontado em um dos artigos é em relação ao perfil feminino estimular mais a criatividade nas organizações do que o masculino. Embora essa pergunta não seja respondida no estudo, há muitas pesquisas interessadas em desvendar se existe diferença entre a liderança feminina e a masculina, como já foi 


\section{A pesquisa sobre Liderança no Brasil: publicações no EnANPAD ao longo de 10 anos}

apontado anteriormente. Outro dado abordado em uma das pesquisas é que os líderes com traços transformacionais conseguem proporcionar um ambiente mais adequado à aprendizagem dos empregados do que aqueles com características transacionais.

Por fim, em relação à liderança política é apontada a dificuldade dos dirigentes dos sindicatos em lidar com o duplo papel que lhe é designado: o de patrão e ao mesmo tempo o de "companheiro". É observado que os sindicatos necessitam de um gerenciamento administrativo, o qual pode tornar mais efetivas as ações no campo político. Como isso não ocorre, a política de "apagar incêndios", em termos gerenciais, é a que predomina. Esse tipo de relação não é muito distante da realidade das atuais organizações, pois o perfil paternalista é comum nas empresas, principalmente nas familiares, o que por vezes, acarreta nessa mesma política de "apagar incêndios" nas empresas.

\section{Outros}

Os artigos que não se encaixaram nos grupos temáticos acima citados foram quatro, e abordaram assuntos como o conceito e o processo de liderança, além da questão do poder. Com o objetivo de explorar as novas abordagens sobre liderança e de contextualizá-la no panorama de transformações organizacionais contemporâneas, os estudos que analisaram o processo de liderança, buscaram verificá-lo como algo complementar, já que o relacionamento dele com os liderados procura uma identificação de ambas as partes, e recíproco, pois o líder almeja ser escolhido e os liderados buscam se reconhecer no líder. Ainda, é ressaltada a importância de estudar o conceito de liderança, bem como a formação de líderes e a maneira como ocorre esse processo, pois assim pode-se facilitar a gestão organizacional, construindo melhores resultados, tanto para a organização, como para os seus membros.

Embora a liderança nas organizações esteja presente não só aos detentores de cargos executivos, nota-se a presença de poder no líder frente aos liderados. Os estudos sobre poder destacam que não há uma conotação positiva ou negativa para tal função, mas sim que há essa relação de poder e liderança, e que a ciência política pode ampliar esse debate. Para isso, são mencionados autores como Maquiavel (1973), Gramsci (1992), Foucault (1995) e Hayek (1977). Os três primeiros autores discorrem acerca da positividade do poder, como condição fundamental, ainda que não exclusiva, para a realização de qualquer projeto na organização, ou seja, o poder tratado como recurso, pois ele é necessário para que se alcancem os objetivos do grupo. Já Hayek (1977) tem uma opinião diferente, e levanta uma questão-chave para o tema do poder na sociedade e, também, nas organizações: como evitar que o poder seja apenas coerção e dominação? Para isso, há de se levar em conta alguns itens determinantes na conduta do líder: a ética e a moral (ABBAGNANO, 1999; HEGEL, 1997). E, por mais que, segundo Maquiavel (1973), a ética por si não dê conta de proteger os grupos da tirania (BIGNOTO, 1992), é preciso que as organizações tenham regras claras para impedir isso.

A complexidade do fenômeno da liderança leva a refletir que há uma necessidade da ocorrência de mais estudos acerca deste tema, que busquem não só um instrumento de avaliação, tampouco a criação de mais um perfil de liderança. É importante que sejam desenvolvidos indicativos que auxiliem no estabelecimento do processo de liderança, não só para gestores, uma vez que a combinação de estratégias e ações coletivas irão influenciar significativamente os resultados organizacionais (AMORIM; AMORIM, 2008).

\section{Considerações Finais}

A pesquisa realizada buscou fazer um levantamento sobre as publicações dos últimos dez anos sobre Liderança em um dos principais eventos da área de Administração no Brasil. O que se pode perceber é que, apesar de muitos autores considerarem este tema muito discutido, os resultados apontam para uma direção em que 0 tema ainda é incipiente e ainda não existe um padrão de pesquisa sobre Liderança entre os pesquisadores brasileiros, demonstrando uma unidade acadêmica como outros países já demonstraram.

CGE * v. $1 *$ n. 1 * Jan-Abr $2013 * 01-16$ 
Os trabalhos encontrados sinalizam poucos assuntos principais e, com isso, pequena tem sido a contribuição para esta temática. Não há inovação nos estudos e também poucos são os trabalhos que aprofundam novas ou já existentes abordagens vinculadas ao tema. Ainda, a grande ocorrência de artigos sobre Liderança em outras áreas, que não em Gestão de Pessoas e Estudos Organizacionais, mesmo que em função dos objetos de estudo escolhidos, demonstra que esse assunto encontra-se disperso e que, com o tempo, pode deixar de ser relacionado a uma área específica como forma de consolidar-se no ambiente da área de Administração.

As abordagens de pesquisa utilizadas indicam que a temática não avançou muito na década analisada. Especialmente considerando o momento de virada de século e inserção de diversas temáticas atuais no cenário de pesquisa. Além disso, o fato de haver em meio à academia aqueles que acreditam ter as teorias de Liderança se esgotado em termos de novas discussões, os resultados desta pesquisa apontam para um debate não muito extenso. Foram 44 artigos em dez anos e as temáticas apresentadas variam em quantidade, porém com pouco volume. Isso faz concluir que não há um aprofundamento em consistência das teorias de Liderança e que, apesar de esse ser um tema central e corrente nas discussões organizacionais, não vem recebendo a devida atenção no meio acadêmico.

Nota-se também que há uma presença forte de literatura estrangeira ligada às pesquisas mencionadas, tanto que dos autores mais citados nos grupos analisados, a maioria foram de estudos realizados fora do País. Talvez essas pesquisas possam ser mais amplas, mas é importante ressaltar que a presença de autores estrangeiros tem influenciado na área e deve ser considerado que as realidades entre países são diferentes. Portanto, há necessidade de mais estudos acerca da Liderança no Brasil envoltos nesse contexto para que haja uma apropriação das teorias no meio acadêmico nacional.

Ainda, na análise detalhada dos artigos publicados sobre Liderança nos últimos dez anos, foi possível perceber que não há uma concentração de autores/instituições que dedicam pesquisas a este tema. Ao mesmo tempo em que isso pode ser positivo, pois traz a sensação de que há diversos grupos engajados nesta temática, trata-se de um dado alarmante, visto que o número de publicações ainda é pequeno (44 artigos publicados no EnANPAD em dez anos) e, portanto, este tema ainda carece de maiores aprofundamentos para que se consiga, quem sabe, alcançar um estado da arte nos estudos sobre Liderança no Brasil.

Um fator que pode ter limitado a quantidade de autores que escrevem sobre Liderança é que para o fim dessa pesquisa só foram utilizados os primeiros autores de cada artigo levantado. Assim, muitos autores que podem ter publicado mais de uma vez na área, não apareceram duplamente por não terem sido os primeiros autores dos artigos. Como sugestão para futuras pesquisas, pode-se ampliar o campo de estudo, buscando levantar os artigos publicados em outros eventos, bem como periódicos relacionados à Administração. Além disso, seria interessante analisar o tema de Liderança sob outras perspectivas, a partir de publicações específicas dos demais campos da Administração, para observar se esse tema também tem espaço em outras áreas.

\section{Referências}

ABBAGNANO, N. Dicionário de filosofia. São Paulo: Martins Fontes, 1999.

AMORIM, T. N. G. F.; AMORIM, A. N. Entre Magos e Magias: como gestores e líderes se efetivam na organização. In: XXXII ENCONTRO DA ANPAD, 32, Rio de Janeiro. Anais... Rio de Janeiro, 2008.

ASSOCIAÇÃO NACIONAL DE PÓS-GRADUAÇÃO E PESQUISA EM ADMINISTRAÇÃO (ANPAD). Rio de Janeiro, 2009. Disponível em http://www.anpad.org.br/. Acesso em: jul. 2009.

AZEVEDO, C. S. Liderança e processos intersubjetivos em organizações públicas de saúde. Ciência e Saúde Coletiva. n. 7 v. 2, 349-361, 2002. 


\section{A pesquisa sobre Liderança no Brasil: publicações no EnANPAD ao longo de 10 anos}

BASS, B. Is there universality in the full range model of leadership? International Journal of Public Administration. New York: v. 19, n. 6; p. 731-762, 1996.

BASS, B. AVOLIO, B. J. Transformational leadership and organizational culture. Public Administration Quarterly. V. 17, n. 1, p. 112-121, 1993.

BARTOL, K. M.; BUTTERFIELD, D. A. Sex effects in evaluating leaders. Journal of Applied Psychology, vol. 61, p446454, 1978.

BASS, B. M.; AVOLIO, B. J. Developing Transformational Leadership: 1992 and Beyond. Journal of European Industrial Training. Bradford: v.14, n.5; p.21-28, 1990.

BASS, B. M.; AVOLIO, B. J. Shatter the glass ceiling: women may make better managers. Human Resource Management. New York: v. 33, n.4, p. 549-561, 1994.

BASS, B. M.; AVOLIO, B. J.Transformational leadership and organizational culture. Public Administration. Quarterly. v.17, n.1, p.112-121, 1993.

BASS, B. M.; AVOLIO, B. J. Transformational leadership and organizational culture. International Journal of Public Administration. New York: v.17, n.3,4; p.541-555, 1995.

BENNIS, W. Líderes: estratégias para assumir a verdadeira liderança. 1988.

BENNIS, W. A invenção de uma vida: reflexões sobre liderança e mudanças. 1 ed. Rio de Janeiro: Campus, 1995; 1996.

BENNIS, W. Managing people is like herding cats. South Provo: Executive Excellence Publishing, 1999a.

BENNIS, W. Old dogs, new tricks. South Provo: Executive Excellence Publishing, 1999b.

BENNIS, W. O futuro da liderança. São Paulo: Futura, 2001.

BENNIS, W.; NANUS, B. Líderes. São Paulo: Harbra, 1988.

BERGAMINI, C. W. Liderança: A administração do sentido. Revista de Administração de Empresa. São Paulo, v. 34, n. 3, p. 102-114, 1994.

BERGAMINI, C. W. Liderança: administração do sentido. São Paulo: Atlas, 1994.

BERGAMINI, C. W. O desafio da liderança. In: BERGAMINI, Cecília W.; CODA, R. Psicodinâmica da vida organizacional: motivação e liderança. 2 ed. São Paulo: Atlas, 1997.

BERGAMINI, C. W. 0 líder eficaz. São Paulo: Atlas, 2002.

BIGNOTO, N. As fronteiras da ética: Maquiavel. In: NOVAES, A. (org.) Ética. São Paulo: Cia.das Letras, 1992.

BRYMAN, A. SCS as a model for research into organizational culture. In: FROST, P. et al. Refraiming organizational culture. Londres: Sage, 1991.

BRYMAN, A. Carisma and Leadership in organizations. London: SAGE Publications, 1992.

BRYMAN, A. Leadership in Organizations In: CLEGG, S. R., HARDY, C. e NORD, W. R. (orgs.). Handbook of organization studies. London: SAGE, 1997, p. 276-292. 
BRYMAN, A. Liderança nas organizações.In: Handbook de Estudos Organizacionais. v.3., p.257-281, São Paulo: Atlas, 2004.

BURNS, J. M. Leadership. New York: Raw Publishers, 1978; 1979.

ENGEN, M. J., LEEDEN R., WILLENSEN, T. Gender, context and leadership styles: a field study. Journal of Occupational and Organizational Psychology. V. 74, p. 581- 598, 2001.

FOUCAULT, M. Microfísica do poder. $11^{\text {a }}$ ed. Rio de Janeiro: Graal, 1995.

GODOI, C. K.; BALSINI, C. P. V. A pesquisa qualitativa nos estudos organizacionais brasileiros: uma análise bibliométrica. In Godoi, C. K.; Mello, R. B.; Silva, A. B. (organizadores.) Pesquisa qualitativa em estudos organizacionais. São Paulo: SARAIVA, 2006.

GORDON, J. L. Creating knowledge maps by exploiting dependent relationships. Knowledge-Based Systems. V. 13, n. 2 , p. $71-79,2000$.

GRAMSCI. A. Obras escolhidas. 2ª ed. São Paulo: Brasiliense, 1992.

HALL, R. H. Organizações: estruturas, processos e resultados. São Paulo: Prentice Hall, 2004.

HAYEK, F. 0 caminho da servidão. $4^{\text {a }}$ ed. Rio: Instituto Liberal, 1987.

HAIR, Joseph F. Jr. Et al. Fundamentos de Pesquisa em Administração. Porto Alegre, Bookman, 2005.

HEGEL, G. W. F. Princípios da filosofia do direito. São Paulo: Martins Fontes, 1997.

HERSEY, P.; BLANCHARD, K. H. Life cycle theory of leadership. Training and Development Journal. V. 23, n. 2, p. 2634, 1969.

HERSEY, P.; BLANCHARD, K. H. Psicologia para administradores: a teoria e as técnicas da liderança situacional. São Paulo: EPU, 1986.

HERSEY, P.; BLANCHARD, K. H. Management of Organizational Behavior: Utilizing Human Resources. Englewood Clifs, NJ. 5a edição, 1988.

KLANN, G.; CARTWRIGHT, T. A question of leadership: Is leadership more an art or more a science? LIA - Leadership in Action: Vol.24, Num.1; p. 12. mar/abr. New Jersey - USA, 2004.

KOTTER, J. Liderando mudança. 12. ed. Rio de Janeiro: Campus,1992.

KOTTER, J. Afinal, o que fazem os líderes. Rio de Janeiro: Campus, 2000.

LE BOTERF, G. Desenvolvendo a Competência dos Profissionais. Porto Alegre: Artmed, 2003.

LIMONGI-FRANÇA, A. C.; ARELLANO, E. B. Liderança, poder e comportamento organizacional. In: As pessoas na organização. São Paulo: Gente, 2002. p. 259-269.

LUIZ, A. J. B. Meta-análise: Definição, aplicações e sinergia com dados espaciais. Cadernos de Ciência \& Tecnologia, Brasilia, v. 19, n. 3, p. 407- 428, set./dez., 2002.

MAQUIAVEL, N. O Príncipe. $1^{\text {a }}$ ed. SP: Abril, 1973. Col. Os Pensadores.

RICKARDS, T.; MOGER, S. Team factors inventory: user's manual. Manchester: Manchester Business School, 1999a.

RICKARDS, T.; MOGER, S. Handbook for creative team leaders. Hampshire: Gower, 1999b.

CGE * v. $1 *$ n. 1 * Jan-Abr 2013 * 01-16 


\section{A pesquisa sobre Liderança no Brasil: publicações no EnANPAD ao longo de 10 anos}

RICKARDS, T.; MOGER, S. Creative leadership processes in project team development: an alternative to Tuckman's model. British Journal of Management, v. 11, n. 4, p. 273-283, Dec. 2000.

SCHEIN, E. H. Organizational Culture and Leadership. San Francisco: Jossey-Bass, 1992.

SCHEIN, E. H. Liderança e cultura organizacional. In: DRUCKER, P. (org). O líder do Futuro. São Paulo: Futura, 1996.

SCHEIN, E. H. What is Culture? In: FROST, MOORE, LOUIS, LUNDBERG, MARTIN. Reframing Organizational Culture. SagePublications, 1991.

ULRICH, D. Organizando de acordo com a capacidade. In: A organização do Futuro. São Paulo: Futura, 1997.

ULRICH, D. Intellectual capital competence x commitment. Sloan Management Review. Cambridge, v.39, n.2, p.15-26, winter, 1998a.

ULRICH, D. Os campeões de recursos humanos. Inovando para obter os melhores resultados. São Paulo: Futura, $1998 \mathrm{~b}$.

ULRICH, D.; ZENGER, J.; SMALLWOOD, N. Liderança orientada para resultados: como os líderes constroem empresas e aumentam a lucratividade. Rio de Janeiro: Campus, 2000.

VENDRAMINI, P. Liderança e mudança organizacional: as categorias essenciais do líder facilitador. Dissertação de Mestrado: UFSC - Florianópolis, 2000.

YULK, G. A. Leadership in organizations. 4th edition. New York: Prentice-Hall Business Publishing, 1989.

YULK, G. A. Liderança Carismática e Transformacional. In: BERGAMINI, Cecília W.; CODA, Roberto (orgs.). Psicodinâmica da Vida Organizacional. São Paulo: Atlas, 1996.

YULK, G. A. Leadership in organizations. 5 ed. New Jersey: Prentice-Hall, 2002.

ZARIFIAN, P. Objetivo Competência. São Paulo: Atlas, 2001. 